\title{
Success rate of methotrexate in the conservative treatment of tubal ectopic pregnancies
}

\author{
CORINA GRIGORIU ${ }^{1,2^{*}}$, ROXANA ELENA BOHILTEA ${ }^{1,2 *}$, BIANCA MARGARETA MIHAI ${ }^{2}$, \\ CORINA AURELIA ZUGRAVU ${ }^{3 *}$, FLORENTINA FURTUNESCU ${ }^{4}$, \\ TIBERIU AUGUSTIN GEORGESCU ${ }^{5}$ and DIANA IONELA MUNTEANU ${ }^{6}$
}

\author{
${ }^{1}$ Department of Obstetrics and Gynecology, 'Carol Davila' University of Medicine and Pharmacy, 020021 Bucharest; \\ ${ }^{2}$ Department of Obstetrics and Gynecology, University Emergency Hospital Bucharest, 050098 Bucharest; \\ ${ }^{3}$ Department of Food Hygiene and Nutrition, 'Carol Davila' University of Medicine and Pharmacy; \\ ${ }^{4}$ Department of Public Health and Management, Faculty of Medicine, 'Carol Davila' University of Medicine and Pharmacy; \\ ${ }^{5}$ Department of Pathology, 'Carol Davila' University of Medicine and Pharmacy, 050463 Bucharest; \\ ${ }^{6}$ Department of Medical Expertise and Work Capacity Recovery, \\ National Institute of Medical Expertise and Work Capacity Recovery, 050653 Bucharest, Romania
}

Received June 23, 2021; Accepted July 23, 2021

DOI: $10.3892 /$ etm.2021.11073

\begin{abstract}
An ectopic pregnancy (EP) involves the implantation of the gestational sac outside the uterine cavity. In the event of diagnosing an EP, the current medical approach is to avoid surgery and to preserve fertility whenever possible; therefore, methotrexate (MTX)-based therapy has become prominent in recent years. MTX, a drug usually used to treat severe forms of autoimmune diseases and several types of cancer, has proven its utility in the conservative treatment of EPs. The success rate of MTX correlates with lower values of $\beta$ subunit of human chorionic gonadotropin hormone ( $\beta$-hCG) serum levels, especially below 2,000 mUI/ml, side effects being insignificant. In the present study, the results obtained concerning the success rate of MTX in the conservative treatment of EPs were obtained at the Department of Obstetrics and Gynecology of the Bucharest University Emergency Hospital from January 2014 to December 2020. The aim of the present study was to highlight the necessity for revising current guidelines for ectopic pregnancy medical treatment in order to manage this pathology optimally and to select carefully the proper treatment, whether medical, surgical or expectant management, so that morbidity is reduced to a minimum.
\end{abstract}

Correspondence to: Professor Roxana Elena Bohiltea, Department of Obstetrics and Gynecology, 'Carol Davila' University of Medicine and Pharmacy, 37 Dionisie Lupu Street, 020021 Bucharest, Romania E-mail: r.bohiltea@yahoo.com

*Contributed equally

Key words: methotrexate, tubal ectopic pregnancy, $\beta$-hCG, conservative treatment, fertility preservation

\section{Introduction}

An ectopic pregnancy (EP) represents the implantation of the gestational sac outside the endometrium of the uterine cavity, and is overall considered a public health problem, judging by the high incidence number reported in recent decades and by its potentially life-threatening medical emergency due to tubal rupture. Previous findings revealed that in the UK the incidence of EP is 11.5 per 1,000 pregnancies and the mortality is 0.4 per 1,000 EP (1): studies from the United States showed the lowest and highest reported incidence, respectively, to be 6.4 and 20.7 per 1,000 EP $(2,3)$. The main risk factors are represented by a previous EP, congenital genital tract anomalies, tumors or history of tubal surgery that distort the normal anatomy, inflammatory pelvic disease, tubal sterilization, combined hormonal contraception or intrauterine device, increasing age, cigarette smoking, assisted reproductive techniques and other medical conditions related to the history and treatment of infertility (4-7). The most common implantation site of an $\mathrm{EP}$ is the fallopian tube (96\%); other anatomic sites are ovary, abdominal cavity, endocervical canal, hysterotomy scar and the myometrium (8).

As a result of technological progress in high-resolution transvaginal ultrasonography and in the sensitive testing of beta subunit of human chorionic gonadotropin hormone $(\beta$-hCG), the EP can be earlier diagnosed, avoiding further complications and improving morbidity and mortality. An early diagnosis of EP allows a more conservative resolution of the pathology. The physician may choose between medical treatment of the EP using methotrexate (MTX), surgical treatment or even expectant management, in selected cases.

The clinical features of EP are represented by a history of amenorrhea, pelvic pain and metrorrhagia (9). An EP may be diagnosed in $90 \%$ of cases by systematic transvaginal examination corroborated with $\beta$-hCG monitoring (10-15). The ultrasonographic diagnosis of an EP is based on 
the visualization outside the uterus of the gestational sac, which contains a yolk sac or embryo with or without cardiac activity (11). The most common finding present in at least $89 \%$ of EP cases is represented by a complex inhomogeneous extraovarian adnexal mass; another suggestive ultrasonographic finding is described as a tubal ring which represents an extraovarian adnexal mass incorporating an empty gestational sac (11). There are also findings that only suggest the presence of an EP, but they are not diagnostic. Endometrial thickness can be an indicator of EP; during normal intrauterine pregnancy the ultrasound shows a mean endometrial thickness of $17.2 \mathrm{~mm}$ versus a mean of $12.7 \mathrm{~mm}$ in EP (15). Douglas and Morrison's pouch should also be inspected for fluid (15). $\beta$-hCG produced by the placental syncytiotrophoblast cells is essential for the diagnosis of viable pregnancy (16-18). Intrauterine viable pregnancy is supported by the tendency of $\beta$-hCG to double every $48-72 \mathrm{~h}$; a suboptimal increase, below $66 \%$, is highly suggestive for an EP (19). A $\beta$-hCG level between 1,000 and 3,500 mUI/ml, and the absence of a gestational sac inside the uterine cavity, highly suggests an EP (16). The differential diagnosis should include miscarriage, implantation bleeding of an intrauterine pregnancy, subchorionic hematoma, gynecologic pathology (ovarian torsion, uterine fibroids, cervical polyps) and gestational trophoblastic disease (19).

When diagnosing a tubal pregnancy (TP), the treatment options depend on the clinical and paraclinical evaluation of the patient, particularly on the hemodynamic status. The treatment options of TP include an expectative approach with monitoring of the $\beta$-hCG value trend, MTX therapy administered intramuscularly, intravenously or injected directly into the gestational sac and surgical treatment consisting of salpingostomy or salpingectomy. The current approach is to avoid surgical treatment and to preserve fertility whenever possible. MTX-based therapy has become increasingly popular in recent years for the medical treatment of $\mathrm{TP}$, along with the close monitoring of the $\beta$-hCG value dynamics $(20,21)$. MTX therapy is also used in combination with uterine artery embolization or supra-selective embolization of the tubal branches of uterine and ovarian arteries for the treatment of cervical pregnancies or caesarean scar pregnancies, the depth of implantation of the gestational sac being dependent on the location of the EP (21-23).

Because of the life-threatening potential, all cases of diagnosed TP must be evaluated in order to establish the need for emergency surgery (hemodynamically unstable patient and/or suspicion of imminent or ongoing tubal rupture). If the case does not require immediate surgery, the next step is the evaluation of the $\beta$-hCG serum level. If the $\beta$-hCG serum value is above $5,000 \mathrm{mIU} / \mathrm{ml}$, surgery is preferred. Furthermore, for patients with lower $\beta$-hCG serum values it is mandatory to evaluate via ultrasonography the presence or absence of embryonic cardiac activity; if present, surgery is preferred $(21,23)$. MTX therapy may be suggested if the embryonic cardiac activity is absent and if there are no contraindications for such therapy including hypersensitivity to MTX, heterotopic pregnancy, breastfeeding, medical conditions such as immunodeficiency, active pulmonary disease or peptic ulcer, or extensive abnormalities in hematologic, hepatic or renal laboratory tests $(24,25)$. Concerning patients with renal insufficiency, MTX having a renal clearance, one dose could cause severe complications including acute respiratory distress, bone marrow suppression, or bowel ischemia $(26,27)$. Finally, the personal preference of the patient should be considered, as the patient should also be perfectly compliant with close monitoring.

The criteria for expectant management of TP include asymptomatic patients, low serum quantitative $\beta$-hCG values ( $<200 \mathrm{mIU} / \mathrm{ml}$ ), pelvic ultrasound with no extra uterine mass and no extra uterine gestational sac, compliant patient with the proposed strategy, understanding the risks and clinical significance of an EP, patients wanting to avoid both surgery or MTX therapy and patients prepared to reach a medical unit if clinical conditions worsened (28).

MTX is usually used as a cytostatic, as well as an antineoplastic, immunosuppressive and anti-inflammatory agent, which inhibits cell proliferation and protein synthesis by suppressing the metabolism of purine bases and nucleic acids. Tetrahydrofolic acid is required in the synthesis of purine bases and nucleic acids and it is produced after the reaction between dehydrofolate reductase and folic acid. MTX is a folic acid analogue, which presents a similar structure and acts as an antagonist by binding to dehydrofolate reductase, leading to lower levels of tetrahydrofolic acid. MTX also affects cells with a high rate of division (29). This characteristic is used in the treatment of EP, active rheumatoid arthritis, non-Hodgkin's lymphoma, breast cancer, cutaneous T-cell lymphoma, gestational trophoblastic disease, desmoid tumors and other neoplastic conditions (30-33).

The evaluation and treatment protocol, after the positive diagnosis of a tubal pregnancy, includes several steps: retreatment laboratory testing, ceasing any administration of folic acid supplements, avoidance of non-steroidal anti-inflammatory drug therapy, as well as physical activity and sexual intercourse, repeated pelvic examinations due to possible tubal rupture, and sun exposure to reduce the risk of MTX dermatitis (26). The next step involves selecting the MTX therapy protocol: single dose, two-dose or multiple-dose protocol. Single-dose protocol suggests a single intramuscular dose of MTX, calculated as $50 \mathrm{mg} / \mathrm{m}^{2}$ of the body surface area (34-37). The $\beta$-hCG serum level is measured on day 1,4 and 7 . If the $\beta$-hCG level decreases less than $15 \%$ between day 4 and 7 , a second dose should be administered $(25,26,38,39)$; this occurs in approximately $15-20 \%$ of cases. Further monitoring involves weekly $\beta$-hCG serum level dosing, until an undetectable level is reached. If the decline of $\beta$-hCG values is not favorable, surgery should be performed. Two-dose protocol includes two MTX doses of $50 \mathrm{mg} / \mathrm{m}^{2}$ offered on day 1 and 4 . The $\beta$-hCG serum level is tested on day 1 and 7 and should decrease more than 15\%; if not, a third dose is administered, followed by retesting on day 11 . If the decrease is less than $15 \%$, a fourth dose may be offered, with retesting on day 14 . If the decline is again not reassuring, surgery should be performed (39). Multiple-dose protocol involves MTX therapy administered on days $1,3,5$ and 7 ( $1 \mathrm{mg} / \mathrm{kg}$ per day, intramuscular) combined with $0.1 \mathrm{mg} / \mathrm{kg}$ leucovorin on days $2,4,6$ and 8 . In the single-dose protocol, leucovorin is not recommended. The $\beta$-hCG level is monitored on days $1,3,5$ and 7 , and this level is expected to decrease more than $15 \%$ from the previous testing. After the treatment is finished, the monitoring phase is initiated by weekly $\beta$-hCG testing until undetectable. 
Thus, the aim of the present study was to highlight the necessity for revising current guidelines for EP medical treatment using MTX, in order to manage this pathology optimally and to select carefully the proper treatment, whether medical, surgical or expectant management, so that morbidity is reduced to a minimum.

\section{Patients and methods}

Patients. We conducted a retrospective study, including 61 women aged between 17 and 46 years diagnosed with EP and treated with MTX at the Department of Obstetrics and Gynecology of the Bucharest University Emergency Hospital, during the period, January 2014-December 2020. The cases were analyzed by collecting data from the Medical Statistics Department of the hospital, without inclusion of personal information. The agreement of the ethics committee (no. 17622/10.04.2018) was required and obtained from the University Emergency Hospital of Bucharest without the need of informed consent for study participation of the patient/legal representative in the case of minors. In total, 509 patients diagnosed with EP were analyzed during the mentioned period, extracting from the database, the medically treated patients, who were advocated in two groups: successful medically treated group (42 patients) and unsuccessful medically treated group (19 patients). Conservatory treated patients were analyzed using clinical and hemodynamic parameters, ultrasound evaluation and a $\beta$-hCG seriated values trend aiming to identify the actual and practical predictive profile of successful medically treated cases of EP.

The inclusion criteria consisted of pregnant patients without evidence of an intrauterine pregnancy on transvaginal ultrasound, associated with visualization of an extrauterine gestational sac with embryo or yolk sac visible on TVUS, plus MTX administration after informed consent was obtained. The exclusion criteria comprised ruptured ectopic pregnancies exclusively surgically treated, renal, pulmonary and liver disease, immunodeficiency, and concomitant intra- and extra-uterine pregnancies.

Methods. Unit protocol for admitted hemodynamic stable patients suspected of EP based on pelvic pain and/or scant brown metrorrhagia, a routine pregnancy test and a transvaginal ultrasound examination, included: clinical observation and caution for pelvic-abdominal pain, metrorrhagia or faintness, blood pressure and pulse monitoring, $\beta$-hCG trend, repeated transvaginal ultrasound, complete blood count, liver and renal tests, blood type and Rh, Human Immunodeficiency and hepatitis $\mathrm{B}$ and $\mathrm{C}$ virus screening. The patients fulfilling the criteria for medical treatment and requiring MTX administration underwent the single- or multiple-dose protocols with further monitoring of the clinical status, ultrasound evolution and $\beta$-hCG seriated values during treatment as well as $\beta$-hCG monitoring after the protocol ended until the $\beta$-hCG serum level became undetectable. All the patients receiving MTX were informed of the risks involved and signed an informed consent form, in order to receive the medication. Patients with $\mathrm{Rh}$ incompatibility received an intramuscular dose of $300 \mathrm{mcg}$ of specific anti-D immunoglobulin. Patients with hemoperitoneum or with decreasing $\beta$-hCG serum levels were excluded from the study. Absolute contraindications of MTX treatment were considered: hemodynamic instability, hemoperitoneum, intrauterine pregnancy, hypersensitivity to MTX, severe medullary depression, active pulmonary pathology, peptic ulcer, severe renal pathology, severe liver pathology, or the impossibility of consistent monitoring of $\beta$-hCG. Patients with contraindications for MTX therapy and those who left the hospital contrary to medical opinion were excluded from the study. The patients with relative contraindications consisting in $\beta$-hCG serum level of over $5,000 \mathrm{mIU} / \mathrm{ml}$, uterine tube diameter $\geq 3.5 \mathrm{~cm}$, present fetal cardiac activity or an embryo sac larger than $4 \mathrm{~cm}$ were accepted for MTX therapy if they refused laparoscopic management as a first line of treatment.

Statistical analysis. The data were collected using Office-Excel version 14.7.7. SPSS 12.0 (IBM Corp.) Pearson's correlation was used for statistical analysis, and the statistical significance threshold was set at $\mathrm{P}<0.05$. The characteristics of each group were calculated using 'Descriptive statistics'.

\section{Results}

During the selected period, 509 patients were diagnosed with extrauterine pregnancy, including 13 patients who were discharged on request or left the hospital contrary to medical advice from the first day of hospitalization, after a single sampling of $\beta$-hCG. Medically treated patients were 61 $(11.98 \%)$. Patient supervision was performed using repeated dosing of $\beta$-hCG serum levels every $48 \mathrm{~h}$ and also by using transvaginal ultrasound. The MTX therapy was successful for 42 patients (68.85\% of cases) representing group 1 of our study, $\beta$-hCG serum levels decreasing during hospitalization (Fig. 1). In 8 cases, a slight increase in the $\beta$-hCG value was observed, mainly consisting of several tens of units in the first $48 \mathrm{~h}$ after the administration of MTX, which led to its subsequent decrease thereafter. The average period of hospitalization was 9 days, with a minimum of 3 and a maximum of 22 days.

In the second group, including 19 cases of 61 medically treated $(31.15 \%)$, MTX therapy failed and surgery was necessary, as the $\beta$-hCG serum level increased or the patients' symptomatology during hospitalization worsened, in most cases complaining of acute pelvic pain. In 3 cases the patients who received MTX were discharged, but returned 10-15 days later to the emergency ward with hemoperitoneum (Fig. 2): one patient was discharged on demand $48 \mathrm{~h}$ after MTX administration, with an insignificant $\beta$-hCG decrease, from 1,908 to $1,846 \mathrm{mIU} / \mathrm{ml}$ (3.24\% decrease), one patient was also discharged on demand after a $48 \mathrm{~h}$ increase in $\beta$-hCG from 3,682 to $3,738 \mathrm{mIU} / \mathrm{ml}$ and another patient was discharged after a decrease in $\beta$-hCG from 13,760 to $4,085 \mathrm{mIU} / \mathrm{ml}$ (total decrease of $70.3 \%$ ). From the 19 cases needing surgery, 9 hospitalized patients including the three patients that were discharged and returned with acute pelvic pain and signs of peritoneal irritation, required emergency surgery as the second line of therapy. In patients where MTX therapy failed and hemoperitoneum was detected, marked increasing of the $\beta$-hCG serum level was observed during repeated determinations, except for the 2 cases described above (Fig. 2). At the time of hemoperitoneum formation in the other patients, the $\beta$-hCG level was at an 


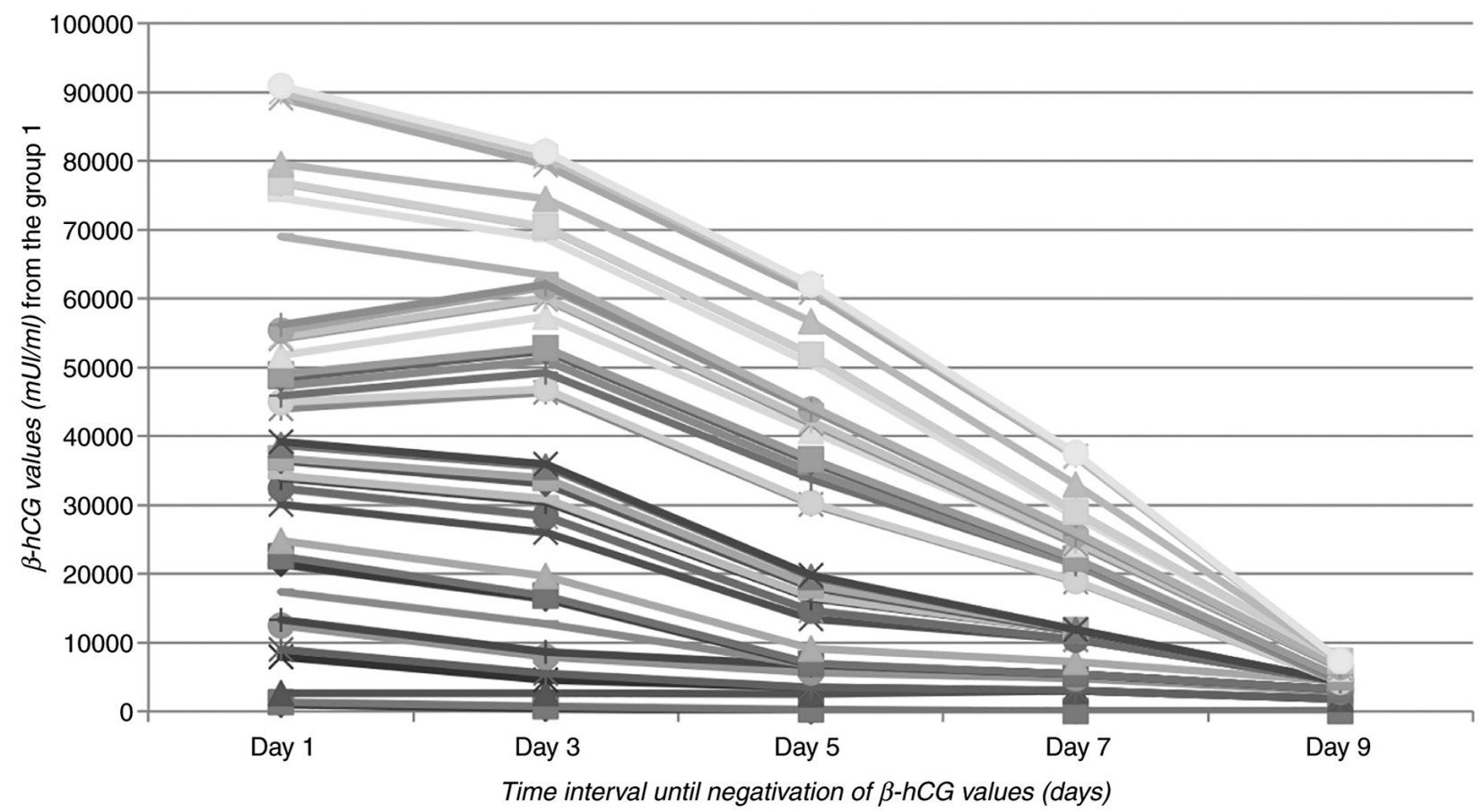

Figure 1. Evolution of $\beta$-hCG values $(\mathrm{mIU} / \mathrm{ml})$ in each patient from group $1(\mathrm{n}=42)$, where the conservative approach was successful. Values show a decreasing trend in all cases, even though the decrease is not always linear and an increase was observed prior to the final decline. Each line represents a patient. The $\mathrm{x}$-axis represents time (days). The y-axis represents $\beta$-hCG values (mIU/ml). $\beta$-hCG, $\beta$ subunit of human chorionic gonadotropin hormone.

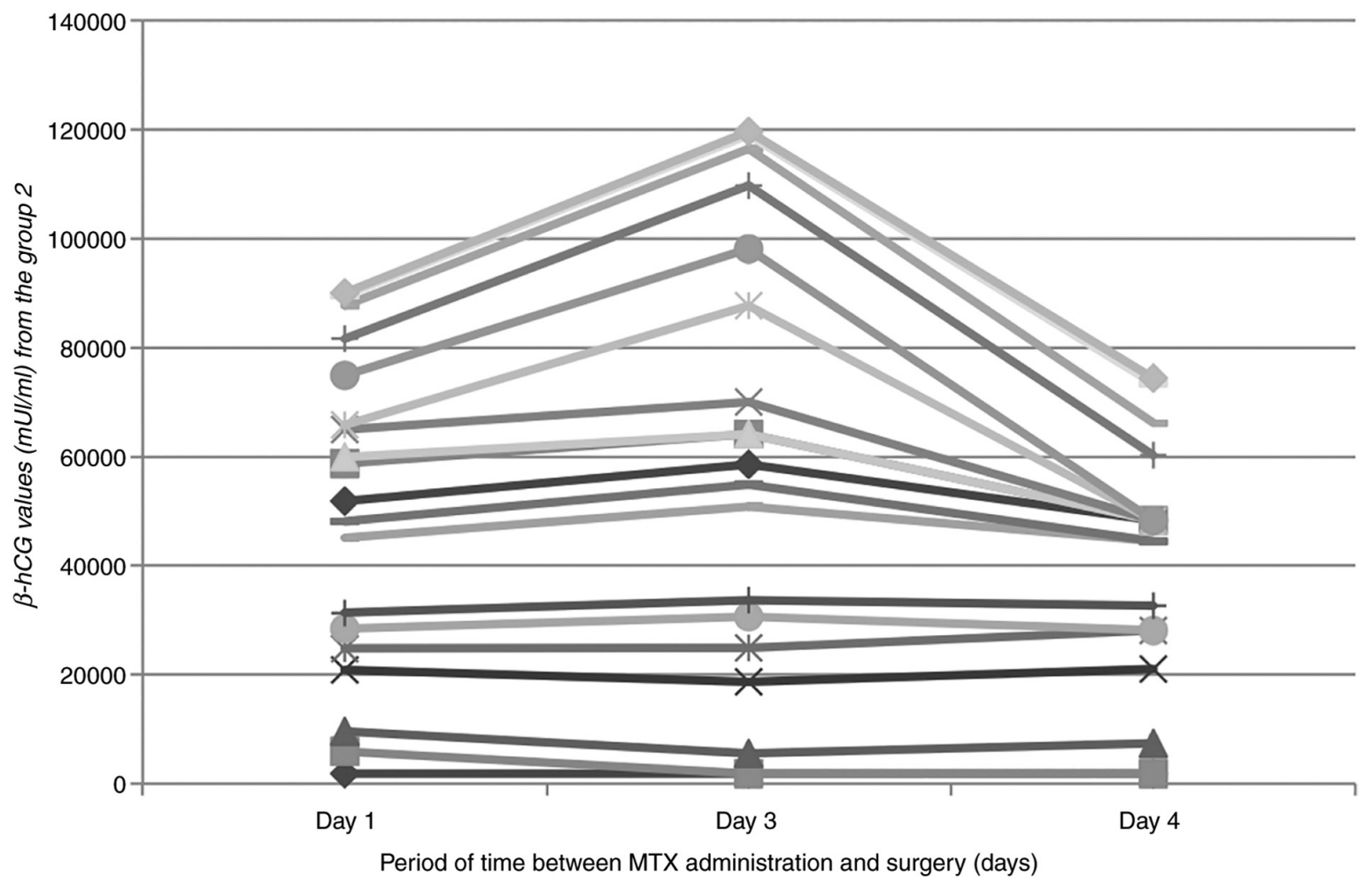

Figure 2. Evolution of $\beta$-hCG values $(\mathrm{mIU} / \mathrm{ml})$ in each patient from group $2(\mathrm{n}=19)$, where the conservative approach failed. Most of the values fail to decrease and/or emergency surgery had to be carried out. Each line represents a patient. The $\mathrm{x}$-axis represents time (days). The y-axis represents $\beta$-hCG values (mIU/ml). $\beta$-hCG, $\beta$ subunit of human chorionic gonadotropin hormone.

average of 4,238 $\mathrm{mIU} / \mathrm{ml}$. In 10 cases, elective surgery was performed, due to the maintenance or increase of the $\beta$-hCG value: 8 patients underwent laparoscopic intervention and 2 patients had an exploratory laparotomy performed. In the group of hemodynamically stable patients, an average $\beta$-hCG value of $7,083 \mathrm{mIU} / \mathrm{ml}$ was detected. Patients with $\beta$-hCG values under $2,000 \mathrm{mIU} / \mathrm{ml}$ had a success rate of $82.7 \%$, statistically significant correlation between the 
initial $\beta$-hCG level $(\mathrm{mIU} / \mathrm{ml})$ and the group in which the patient was included ( $\mathrm{P}=0.003)$ (Fig. 1). The failures of medical therapy required emergency surgery $(14.75 \%)$ and elective surgery $(16.39 \%)$.

\section{Discussion}

There are several studies revealing the success rate of a single dose administration of MTX up to 94\%, the administration of a single intramuscular dose being better tolerated by the patient and better assimilated rather than the sequence of administration of smaller, serial doses $(34,35,37-40)$.

The success rate of MTX is lower when embryonic heartbeats are detected, correlating with a higher $\beta$-hCG serum level (41). The most important factor regarding the prediction of the MTX therapy success rate represents the initial value of the $\beta$-hCG level (42). In our study, patients with values $<2,000 \mathrm{mIU} / \mathrm{ml}$ showed the best response, with higher values positively correlated with being in-group 2 , rather than in-group 1 . There was no statistically significant correlation between the age of the patients and the success of the conservative therapy.

In the present study, the failure rate of MTX treatment was $31.15 \%$, with almost equal percentages of patients requiring emergency surgery and elective surgery due to increased $\beta$-hCG levels, even when two doses of MTX were administered (difference not statistically significant). The high failure rate compared to other studies was correlated with the $\beta$-hCG value measured before starting the treatment, which depended on the time frame of when the patient presented to the hospital. Patients usually presented to the hospital when pelvic pain occurred and required immediate care. MTX therapy was also attempted in patients presenting higher $\beta$-hCG values, the success rate being of $50 \%$ in patients with $\beta$-hCG values above $4,000 \mathrm{mIU} / \mathrm{ml}$ at the moment of the healthcare unit admission.

Worldwide, the inpatient hospital treatment of EP has decreased while multiple health care visits for a single EP have increased. Taking into account that after MTX administration, rigorous patient supervision is fundamental, with $\beta$-hCG monitoring, clinical and hemodynamic parameters monitoring, and any complaints of abdominal-pelvic pain, Kehr's sign (acute pain in the tip of the shoulder), abnormal uterine bleeding, faintness, nausea, tachycardia and blood pressure decrease should raise the suspicion of intraperitoneal bleeding, we strongly recommend that each country to adapt the management of surveillance of medically treated EP cases upon the real promptitude and performance of its own healthcare system. Due to the lack of specific guidance, low level of medical education of some patients and the deficiencies in informational system of the medical care, inpatient hospital treatment of ectopic pregnancy until the $\beta$-hCG value has decreased to under the life-threatening level is optimal.

MTX consistently targets rapidly dividing cells, such as gastrointestinal tract cells, epidermal and hematopoietic cells, which are most commonly affected. The side effects of MTX administration consist of nausea, intestinal transit disorders, fatigue, abdominal pain, pancytopenia, increased risk of infections and dermatitis (43). High elevated serum liver enzymes, chronic liver injury, cirrhosis and portal hypertension may occur when given in high doses. As a result of cellular destruction, an elevated uric acid concentration in patients receiving MTX is expected. Breastfeeding is contraindicated because MTX is distributed in breast milk (33). Due to the hepatotoxic effects, it is imperative to screen for chronic viral hepatitis $\mathrm{B}$ and $\mathrm{C}$ and to recommend reducing alcohol consumption to a minimum in the immediate period following MTX administration. The patient should be counseled to avoid excessive exposure to sunlight and UV lamps for a period of 4 weeks, as there have been recorded cases of dermatitis. Due to the risk of pancytopenia and the increased risk of infection, we recommend the use of external menstrual pads and the avoidance of intravaginal tampons in case of spotting or vaginal bleeding.

In conclusion, the advantage of using MTX in extrauterine pregnancies consists in surgery avoidance, with the possibility of maintaining fertility and the prevention of long-term complications of surgery such as adhesions that may cause chronic pain and modify the quality of life. Medical management of EP should be considered whenever a patient meets the criteria. The success rate of MTX is correlated with lower initial $\beta$-hCG serum levels, especially below 2,000 $\mathrm{mIU} / \mathrm{ml}$; MTX therapy efficiency decreases with higher $\beta$-hCG serum values. At present, the suggested superior cut-off $\beta$-hCG value proposed is $5,000 \mathrm{mIU} / \mathrm{ml}$ for the medical treatment of EP in the majority of international guidelines. Given our 50\% success rate for ectopic pregnancies with $\beta$-hCG values over $4,000 \mathrm{mIU} / \mathrm{ml}$, we recommend revising the actual criteria, thereby decreasing the superior cut-off value for optimal future results. With the introduction of MTX therapy, the need for surgery has been significantly reduced, sparing the unnecessary use of resources, avoiding possible surgical complications, providing comfort to the patients and reducing morbidity caused by the surgery. In addition, medical therapy is less invasive, has a diminished cost and presents a similar intrauterine pregnancy rate for future pregnancies.

\section{Acknowledgements}

Not applicable.

\section{Funding}

No funding was received.

\section{Availability of data and materials}

Any additional information concerning the study can be requested from the corresponding author on reasonable request.

\section{Authors' contributions}

CG, REB, and BMM conceived the article and wrote the draft of manuscript. CG, DIM and TAG collected, analyzed and interpreted the patient data regarding the methotrexate administration for the treatment of ectopic pregnancy. REB, TAG and BMM performed the literature search and conducted the follow-up of the patients. CAZ and FF performed statistical analysis and study description, making a substantial 
contribution to the conception of the work and interpretation of data. CG, REB and DIM revised the manuscript critically for important intellectual content. The authenticity of all the raw data was assessed by CG, FF and DIM. All authors have read and approved the final manuscript.

\section{Ethics approval and consent to participate}

The present study was conducted in accordance with the World Medical Association Declaration of Helsinki. The data collected retrospectively did not contain personal information and only the Ethics Committee agreement no. 17622/10.04.2018 of the University Emergency Hospital of Bucharest was required and obtained without the need of informed consent or the consent of the patient/legal representative in the case of minors.

\section{Patient consent for publication}

Not applicable.

\section{Competing interests}

The authors declare that they have no competing interests.

\section{References}

1. Tay JI, Moore $\mathrm{J}$ and Walker JJ: Ectopic pregnancy. West J Med 173: 131-134, 2000.

2. Van Den Eeden SK, Shan J, Bruce C and Glasser M: Ectopic pregnancy rate and treatment utilization in a large managed care organization. Obstet Gynecol 105: 1052-1057, 2005.

3. Hoover KW, Tao G and Kent CK: Trends in the diagnosis and treatment of ectopic pregnancy in the United States. Obstet Gynecol 115: 495-502, 2010.

4. Bouyer J, Coste J, Shojaei T, Pouly JL, Fernandez H, Gerbaud L and Job-Spira N: Risk factors for ectopic pregnancy: A comprehensive analysis based on a large case-control, population-based study in France. Am J Epidemiol 157: 185-194, 2003.

5. Malacova E, Kemp A, Hart R, Jama-Alol K and Preen DB: Long-term risk of ectopic pregnancy varies by method of tubal sterilization: A whole-population study. Fertil Steril 101: 728-734, 2014.

6. Nybo Andersen AM, Wohlfahrt J, Christens P, Olsen J and Melbye M: Maternal age and fetal loss: Population based register linkage study. BMJ 320: 1708-1712, 2000.

7. Bohîltea RE, Zugravu CA, Neacsu A, Navolan D, Berceanu C, Nemescu D, Bodean O, Turcan N, Baros Al and Cîrstoiu MM: The prevalence of Vitamin D deficiency and its obstetrical effects. A prospective study on Romanian patients. Rev Chim 70: 1228-1233, 2019.

8. Bouyer J, Coste J, Fernandez H, Pouly JL and Job-Spira N: Sites of ectopic pregnancy: A 10 year population-based study of 1800 cases. Hum Reprod 17: 3224-3230, 2002.

9. Belics Z, Gérecz B and Csákány MG: Early diagnosis of ectopic pregnancy. Orv Hetil 155: 1158-1166, 2014 (In Hungarian).

10. Van Mello NM, Mol F, Ankum WM, Mol BW, Van der Veen F and Hajenius PJ: Ectopic pregnancy: How the diagnostic and therapeutic management has changed. Fertil Steril 98: 1066-1073, 2012 .

11. Webster K, Eadon H, Fishburn S and Kumar G; Guideline Committee: Ectopic pregnancy and miscarriage: Diagnosis and initial management: Summary of updated NICE guidance. BMJ 367: I6283, 2019.

12. Atri M, Leduc C, Gillett P, Bret PM, Reinhold C, Kintzen G, Aldis AE and Thibodeau M: Role of endovaginal sonography in the diagnosis and management of ectopic pregnancy. Radigraphics 16: 755-774; discussion 775, 1996.

13. Dialani V and Levine D: Ectopic pregnancy: A review. Ultrasound Q 20: 105-117, 2004.

14. Winder S, Reid S and Condous G: Ultrasound diagnosis of ectopic pregnancy. Australas J Ultrasound Med 14: 29-33, 2011.
15. Moschos E and Twickler DM: Endometrial thickness predicts intrauterine pregnancy in patients with pregnancy of unknown location. Ultrasound Obstet Gynecol 32: 929-934, 2008.

16. Surampudi K and Gundabattula SR: The role of serum beta hCG in early diagnosis and management strategy of ectopic pregnancy. J Clin Diagn Res 10: QC08-QC10, 2016.

17. Cole LA: Individual deviations in human chorionic gonadotropin concentrations during pregnancy. Am J Obstet Gynecol 204: 349. e1-7, 2011.

18. Seeber BE: What serial hCG can tell you, and cannot tell you, about an early pregnancy. Fertil Steril 98: 1074-1077, 2012.

19. Dogra V, Paspulati RM and Bhatt S: First trimester bleeding evaluation. Ultrasound Q 21: 69-85; quiz 149-50, 153-4, 2005.

20. Van Mello NM, Mol F, Adriaanse AH, Boss EA, Dijkman AB, Doornbos JP, Emanuel MH, Friederich J, Leeuw-Harmsen Lv, Lips JP, et al: The METEX study: Methotrexate versus expectant management in women with ectopic pregnancy: A randomised controlled trial. BMC Women's Health 8: 10 2008.

21. Kirk E, Condous G, Haider Z, Syed A, Ojha K and Bourne T: The conservative management of cervical ectopic pregnancies. Ultrasound Obstet Gynecol 27: 430-437, 2006.

22. Mesogitis S, Pilalis A, Daskalakis G, Papantoniou N and Antsaklis A: Management of early viable cervical pregnancy. BJOG 112: 409-411, 2005.

23. Timor-Tritsch IE, Monteagudo A, Santos R, Tsymbal T, Pineda G and Arslan AA: The diagnosis, treatment, and follow-up of cesarean scar pregnancy. Am J Obstet Gynecol 207: 44, e1-13, 2012.

24. American College of Obstetricians and Gynecologists: ACOG Practice Bulletin No. 94: Medical management of ectopic pregnancy. Obstet Gynecol 111: 1479-1485, 2008.

25. Practice Committee of American Society for Reproductive Medicine: Medical treatment of ectopic pregnancy: A committee opinion. Fertil Steril 100: 638-644, 2013.

26. Kelly H, Harvey D and Moll S: A cautionary tale: Fatal outcome of methotrexate therapy given for management of ectopic pregnancy. Obstet Gynecol 107: 439-441, 2006.

27. Teal SB: A cautionary tale: Fatal outcome of methotrexate therapy given for management of ectopic pregnancy. Obstet Gynecol 107: 1420-1; author reply 1421, 2006.

28. Korhonen J, Stenman UH and Ylöstalo P: Low-dose oral methotrexate with expectant management of ectopic pregnancy. Obstet Gynecol 88: 775-778, 1996.

29. Bleyer WA: The clinical pharmacology of methotrexate: New applications of an old drug. Cancer 41: 36-51, 1978.

30. Kremer JM, Lawrence DA, Hamilton R and McInnes IB: Long-term study of the impact of methotrexate on serum cytokines and lymphocyte subsets in patients with active rheumatoid arthritis: Correlation with pharmacokinetic measures. RMD Open 2: e000287, 2016.

31. Moura CS, Schieir O, Valois MF, Thorne C, Bartlett SJ, Pope JE, Hitchon CA, Boire G, Haraoui B, Hazlewood GS, et al: Treatment strategies in early rheumatoid arthritis methotrexate management: Results from a prospective cohort. Arthritis Care Res (Hoboken) 72: 1104-1111, 2020.

32. National Comprehensive Cancer Network (NCCN). NCCN clinical practice guidelines in oncology. https://www.ncen. org/professionals/physician_gls (Accessed on August 19, 2021).

33. National Center for Biotechnology Information. PubChem Database. Methotrexate, 2021 https://pubchem.ncbi.nlm.nih. gov/compound/Methotrexate (Accessed on 14 March 2021).

34. Lipscomb GH, Bran D, McCord ML, Portera JC and Ling FW: Analysis of three hundred fifteen ectopic pregnancies treated with single-dose methotrexate. Am J Obstet Gynecol 178: 1354-1358, 1998.

35. Barnhart KT, Gosman G, Ashby R and Sammel M: The medical management of ectopic pregnancy: A meta-analysis comparing 'single dose' and 'multidose' regimens. Obstet Gynecol 101: 778-784, 2003.

36. Morlock RJ, Lafata JE and Eisenstein D: Cost-effectiveness of single-dose methotrexate compared with laparoscopic treatment of ectopic pregnancy. Obstet Gynecol 95: 407-412, 2000.

37. Lipscomb GH: Medical therapy for ectopic pregnancy. Semin Reprod Med 25: 93-98, 2007

38. Stovall TG, Ling FW, Gray LA, Carson SA and Buster JE: Methotrexate treatment of unruptured ectopic pregnancy: A report of 100 cases. Obstet Gynecol 77: 749-753, 1991. 
39. Kirk E, Condous G, Van Calster B, Haider Z, Van Huffel S, Timmerman D and Bourne T: A validation of the most commonly used protocol to predict the success of single-dose methotrexate in the treatment of ectopic pregnancy. Hum Reprod 22: 858-863, 2007. 40. Lipscomb GH, Stovall TG and Ling FW: Nonsurgical treatment of ectopic pregnancy. N Engl J Med 343: 1325-1329, 2000.

41. Erdem M, Erdem A, Arslan M, Oç A, Biberoglu K and Gursoy R: Single-dose methotrexate for the treatment of unruptured ectopic pregnancy. Arch Gynecol Obstet 270: 201-204, 2004.
42. Liposcomb GH, McCord ML, Stovall TG, Huff G, Portera SG and Ling TW: Predictors of success of methotrexate treatment in women with tubal ectopic pregnancies. N Engl J Med 341: 1974-1978, 1999.

43. Alur-Gupta S, Cooney LG, Senapati S, Sammel MD and Banhart KT: Two dose versus single dose of methotrexate for treatment of ectopic pregnancy: A meta-analysis. Am J Obstet Gynecol 221: 95-108. e2, 2019. 